\title{
Selective surface/interface characterization of thin garnet films by magnetization-induced second-harmonic generation
}

\author{
Fredrik Hansteen, ${ }^{1,2, *}$ Ola Hunderi, ${ }^{2}$ Tom Henning Johansen, ${ }^{3}$ Andrei Kirilyuk, ${ }^{1}$ and Theo Rasing ${ }^{1}$ \\ 'NSRIM, University' of Nijmegen, Toernooiveld 1, 6525 ED Nijmegen, The Netherlands \\ ${ }^{2}$ The Norwegian University of Science and Technology, 7491 Trondheim, Norway \\ ${ }^{3}$ Depariment of Physics, University of Oslo, P. O. Box 1048, Blindern, Norway' \\ (Received 2 December 2003; revised manuscript received 27 April 2004; published 10 September 2004)
}

\begin{abstract}
Magnetization-induced second-harmonic generation (SHG) has been used for the study of thin garnet films. The strong optical absorption of these films at the second harmonic frequency provides a unique possibility to study magnetic and structural properties of the film surface and film/substrate interface separately. When studicd in transmission at normal incidence all relevant elements of the crystallographic part of the nonlinear optical susceptibility tensor vanish identically, while a magnetization-induced contribution remains. The purely magnetic origin of SHG from the interface is unambiguously demonstrated. From measurements of the SHG rotational anisotropy and magnetic field dependence, the surface is found to have inclusions which act as nonmagnetic sources of SHG, while the anisotropy causing easy in-plane magnetization is found to be stronger at the interface with the substrate than at the surface of the film.
\end{abstract}

DOI: $10.1103 /$ PhysRevB.70.094408

PACS number(s): 75.50.Gg, 78.20.Ls, 42.65.Ky, 75.70.-i

\section{INTRODUCTION}

Ferrimagnetic garnets constitute an interesting group of transparent magnetic materials with large linear magnetooptical (MO) effects that make them very attractive for technological applications. ${ }^{1}$ They can accept a wide range of dopants which alter their properties in various ways, and were therefore studied extensively over the past 50 years. ${ }^{1,2}$ Optical components such as modulators, switches, and isolators based on garnets have already found widespread use.

Recently there has been significant interest in thin garnet films as sensors and indicators for magnetic fields. Garnet films with in-plane magnetization have been found to be exceptionally well suited for magnetic field visualization and imaging (see Refs. 3 and 4, and references therein). Very little domain activity and a continuous rotation of the magnetization vector in response to an increasing external field are some of their favorable properties. Films of this type have successfully been used for imaging magnetic domains, recorded patterns in magnetic storage media, currents in microelectronic circuits, and for the study of defects, currents, and vortices in superconductors. ${ }^{5-7}$

Garnet films are most commonly grown by liquid phase epitaxy (LPE), but also pulsed laser deposition, gel methods, and other techniques are being used. For imaging applications it is particularly important to have high quality, uniform, single-domain films with high sensitivity and little defects. These are properties which can be tailored to a large degree by substitution and by tuning of the growth conditions. It is, for instance, well known that Bismuthsubstitution on dodecahedral sites of the garnet crystal strongly enhances the linear magneto-optical response. Furthermore, the film and substrate compositions influence the lattice matching at the interface. A small mismatch will cause strain in the grown film, while larger values of the lattice mismatch may, in addition, cause growth of differently ori- ented crystallites, defects in the crystal structure, or even cracking of the film or substrate. A small lattice mismatch is sometimes deliberately introduced in order to obtain the desired magnetic anisotropy and easy direction of magnetization. For garnet films grown on (100) oriented gadolinium gallium garnet (GGG) substrates the strain is uniaxial, and will, depending on the sign of the anisotropy constant, strive to align the spontaneous magnetization either along the anisotropy axis (easy-axis) or normal to this axis (easy-plane). The dominant factor in deciding the easy direction of magnetization will, however, often be the shape anisotropy.

The sensitivity of the MO response of garnet films to an extemal field is one of the main properties of interest for imaging applications. It is commonly investigated by measuring the external field dependence of the Faraday rotation in the spectral region of interest. This, however, reflects only averaged bulk properties of the film without addressing the possibility that there could be a significant inhomogeneity across the thickness of the film. Relaxation of interfacial strain due to lattice mismatch may cause different anisotropy fields and thus different MO sensitivity for the film surface and interface regions. A technique that is sensitive to magnetization and capable of distinguishing between surface and interface is needed in order to study these effects.

Magnetization induced second harmonic generation is a nonlinear optical technique which has a particular sensitivity to surfaces and interfaces of magnetic materials. In the electric dipole approximation nonlinear optical effects of even order, such as second harmonic generation (SHG), are forbidden in the bulk of centrosymmetric media but are allowed at interfaces where the centrosymmetry is lifted. ${ }^{8}$ Magnetooptical effects arise due to the breaking of time reversal symmetry by a spontaneous or induced magnetization. Hence, nonlinear magneto-optical effects occur only in media where the time reversal and space reversal inversion symmetry are broken simultaneously. This has caused magnetization in- 
duced second harmonic generation (MSHG) to become an important tool for studying surfaces and buried interfaces of magnetic materials, ${ }^{9-14}$ including antiferromagnetic systems. ${ }^{15,16}$ Previously, an analysis of SHG in garnet films of different classes of symmetry has been given by Pisarev et al. ${ }^{17}$ Gridnev et al. ${ }^{18}$ and Pavlov. ${ }^{19}$

In this paper we report on an experimental study of SHG in garnet films with in-plane magnetization, grown for the purpose of MO imaging by liquid phase epitaxy on (100) oriented substrates. We have chosen the experimental geometry so that crystallographic contributions to the SHG should vanish completely, leaving only magnetization-induced SHG. Despite a broken space inversion symmetry in these films, the surface and interface regions may be studied independently. This is made possible by a strong optical absorption in the films at the second harmonic frequency, leading to a escape depth of about $0.2 \mu \mathrm{m}$ for light at $2 \omega$, and provides a very powerful tool for the characterization of the interfaces of these materials.

\section{THEORY}

The induced polarization $\mathbf{P}$ in a medium due to an incident electromagnetic wave $\mathbf{E}$ can be written in the electric dipole approximation as an expansion in powers of the electric field of the incident wave

$$
\mathbf{P}=\epsilon_{0}\left(\chi^{(1)} \mathbf{E}+\chi^{(2)} \mathbf{E E}+\ldots\right) .
$$

The coefficients $\chi^{(n)}$ are the frequency dependent electric susceptibilities of order $n$. The first order susceptibility gives rise to the linear optical response, while the higher order terms give rise to nonlinear optical effects that become observable for strong incident fields. In the presence of a magnetization $\mathrm{M}$, each of the susceptibilities $\chi^{(n)}$ should be further expanded in powers of $\mathbf{M}$

$$
\chi^{(n)}=\chi_{0}^{(n)}+\frac{\delta \chi^{(n)}}{\delta \mathbf{M}} \mathbf{M}+\ldots
$$

The first term, independent of $\mathbf{M}$, can be thought of as the purely crystallographic contribution, while the higher order terms may only exist in the presence of a magnetization. These give rise to a magnetic induced contribution. In the following we consider only contributions up to first order in M.

In a local frame of reference, the second order nonlinear polarization at frequency $2 \omega$ induced by an incident field $e$ of frequency $\omega$ in a medium with magnetization $m$ can be written as

$$
p_{i}^{2 \omega}=\epsilon_{0} \chi_{i j k}^{(\mathrm{cr})} e_{j}^{\omega} e_{k}^{\omega}+\epsilon_{0} \chi_{i j k l}^{(m)} e_{j}^{\omega} e_{k}^{\omega} m_{l},
$$

where $\{i, j, k, l\}=\{x, y, z\}$. Here $\chi_{i j k}^{(\mathrm{cr})}$ is a third rank polar tensor representing the crystallographic contribution, and $\chi_{i j k l}^{(m)}$ is a fourth rank axial tensor representing the magnetization induced contribution. The symmetry of the medium imposes restrictions on the susceptibility tensors according to Neumann's principle, thereby reducing the number of independent tensor components.

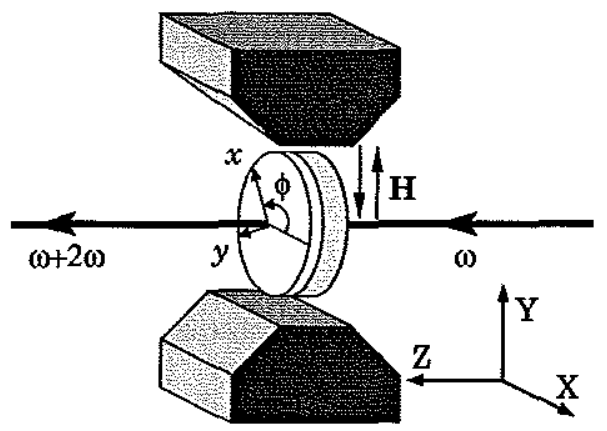

FIG. 1. Experimental setup. The sample, a thin garnet film on a GGG substrate, is illuminated by femtosecond laser pulses and rotated about an axis normal to the film plane in a magnetic field along the $\pm Y$-direction. The angle $\phi$ denotes the rotation of the sample's $x$-axis with respect to the $X$-axis of the laboratory system of reference.

The observable intensity of radiated light at the second harmonic frequency is given by the square of the induced polarization at $2 \omega$

$$
\begin{aligned}
I(2 \omega) \propto & |\mathrm{P}(2 \omega)|^{2} \propto\left|\chi^{(\mathrm{cr})}+\chi^{(m)}\right|^{2} \propto\left|\chi^{(\mathrm{cr})}\right|^{2}+\left|\chi^{(m)}\right|^{2} \\
& +2\left|\chi^{(\mathrm{cr})}\right|\left|\chi^{(m)}\right| \cos \Delta \Phi .
\end{aligned}
$$

A phase difference $\Delta \Phi$ between $\chi^{(\mathrm{cr})}$ and $\chi^{(m)}$ different from $\pi / 2$ will lead to interference between crystallographic and magnetic contributions. Information about the phase is generally lost when measuring intensities, but may be recovered using a technique based on interference with an external reference. ${ }^{20}$

Bulk garnet crystals have cubic symmetry and are centrosymmetric, thereby limiting any SHG to a very thin region near the surface or interface where the space inversion symmetry is broken. In thin garnet films, however, the inversion symmetry appears to be broken ${ }^{18,19}$ due to what might be a growth induced distortion of the symmetry along the direction of growth. This reduces the symmetry of our thin film to the tetragonal $4 \mathrm{~mm}$ point group, and allows for crystallographic bulk contributions to the SHG. In the presence of a magnetization that causes breaking of the time-inversion symmetry, also magnetization-induced contributions from the bulk will be allowed.

Now, consider a crystalline magnetic thin film of point group symmetry $4 \mathrm{~mm}$ illuminated at normal incidence along the $z$-direction, and exposed to a transverse magnetic field, as shown in Fig. 1. In this geometry all elements of $\chi^{(\mathrm{cr})}$, the crystallographic part of the second order susceptibility tensor in Eq. (3), vanish identically, while the nonzero components of $\chi_{i j k l}^{(m)}$ are $^{18,21}$

$$
\begin{gathered}
A=\chi_{x x x y}=-\chi_{y y y x}, \\
B=\chi_{x x y x}=\chi_{x y x x}=-\chi_{y x y y}=-\chi_{y y x y}, \\
C=\chi_{y x x x}=-\chi_{x y y y} .
\end{gathered}
$$

Both crystal symmetry and experimental geometry determine which tensor components contribute to the observable SHG signal. At non-normal incidence also crystallographic contri- 
butions would be present for media belonging to the $4 \mathrm{~mm}$ point group.

When the crystalline film is rotated an angle $\phi$ about the $Z$-axis and exposed to a stationary magnetic field along the $Y$-axis, the induced polarization at frequency $2 \omega$ in the stationary $X Y Z$ frame of reference can readily be deduced

$$
\begin{gathered}
P_{X X}=M E_{X}^{2} \frac{\epsilon_{0}}{4}[(3 A-2 B-C)+(A+2 B+C) \cos 4 \phi] \\
P_{X Y}=M E_{X}^{2} \frac{\epsilon_{0}}{4}[(A+2 B+C) \sin 4 \phi] \\
P_{Y X}=M E_{Y}^{2} \frac{\epsilon_{0}}{4}[(A+2 B-3 C)-(A+2 B+C) \cos 4 \phi] \\
P_{Y Y}=-M E_{Y}^{2} \frac{\epsilon_{0}}{4}[(A+2 B+C) \sin 4 \phi] .
\end{gathered}
$$

$P_{I J}$ denotes the induced polarization along the $J$ direction due to an incident wave polarized along the $I$ direction.

For the case of $4 \mathrm{~mm}$ symmetry, as can be seen from Eqs. (4) and (6)-(9), the radiated intensity $I(2 \omega)$ is quadratic in $\mathbf{M}$, and is therefore not expected to change upon reversal of the direction of the magnetization, $\mathbf{M} \rightarrow-\mathbf{M}$.

If the aforementioned experimental geometry is modified to magnetize the films in the out-of-plane $Z$-direction, then also $\chi^{(m)} \equiv 0$ for symmetry reasons and no SHG will take place.

\section{EXPERIMENTAL PROCEDURE}

An approximately $3 \mu \mathrm{m}$ thick garnet film of composition $\mathrm{Lu}_{2.5} \mathrm{Bi}_{0.5} \mathrm{Fe}_{5} \mathrm{O}_{12}$, grown on a (100) oriented gadolinium gallium garnet (GGG) substrate was illuminated at normal incidence by femtosecond laser pulses of $\lambda=840 \mathrm{~nm}$ generated by a Ti:Sapphire laser system. The incident beam was focused to a spot size of approximately $200 \mu \mathrm{m}$ on the sample, which was exposed to a magnetic field in the $Y$-direction, as shown in Fig. 1. The transmitted second harmonic intensity was detected using a standard photon counting technique involving a cooled photomultiplier tube (PMT), an amplifier, and counting electronics. The spontaneously in-plane magnetized garnet film was exposed to a stationary magnetic field to keep the magnetization fixed along the $Y$-axis as the sample was rotated.

The polarization of the incident wave was set using a Babinet-Soleil compensator followed by a Glan-Taylor polarizer. An edge filter (RG640) was used to exclude the possibility of SH light generated in any of the optical components reaching the sample and influencing the measurements. Similarly, a band-pass filter (BG39) was placed immediately after the sample to filter out the strong fundamental beam. An additional filter was used to absorb the strong two-photon luminescence at $\lambda=520-560 \mathrm{~nm}$ exhibited by the samples under study. An analyzer, also of the Glan-Taylor type, was placed before the photomultiplier and used to determine the polarization of the SH light.

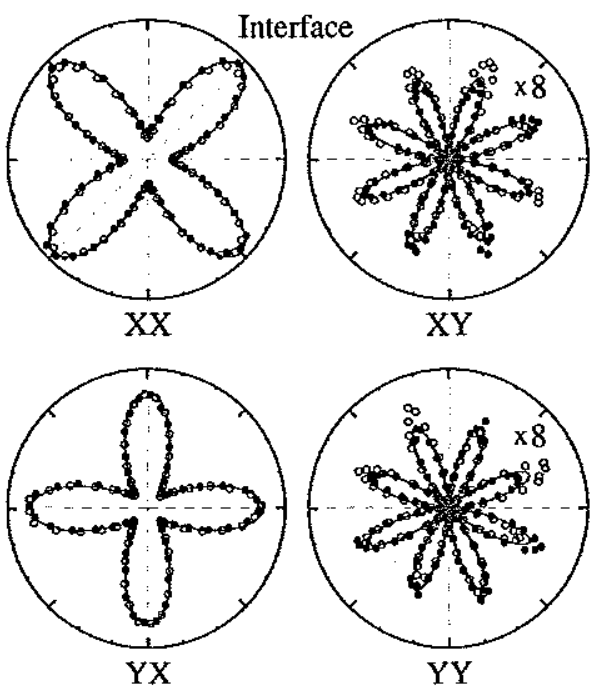

FIG. 2. Polar plots of the measured SHG rotational anisotropy $I(\phi)$ originating from the film-substrate interface for a full $360^{\circ}$ azimuthal rotation of the sample in a transverse magnetic field. The symbols $\{X, Y\}$ below each plot denote the settings of polarizer and analyzer transmission axis, respectively. Open and filled circles represent measurements with the magnetic freld in the $+Y$ and $-Y$ direction. The solid lines represent best fits to the data points.

Rotational anisotropy measurements of the SHG were made by recording the intensity of light at $2 \omega$ as a function of the sample angle of rotation $\phi$ about an axis normal to the film plane. This was done for light incident both from the film and substrate side of the sample and for different combinations of polarizer and analyzer settings.

The GGG material used as a substrate is essentially transparent both to the fundamental beam at $\lambda=840 \mathrm{~nm}$ and to the second harmonic. ${ }^{22}$ The magnetic garnet film also exhibits low linear optical absorption at the fundamental wavelength, but has a very strong absorption at the second harmonic frequency of about $\alpha=5 \times 10^{4} \mathrm{~cm}^{-1}(\epsilon \approx 7+i)$. The linear optical properties of a series of garnet films, the ones studied here included, have previously been obtained from ellipsometric measurements. ${ }^{23}$ The strong absorption of the second harmonic wave makes it possible to study the film surface and interface separately by illuminating it from either the substrate or surface side, respectively. Since the escape depth of light at $2 \omega$ is approximately $0.2 \mu \mathrm{m}$, only a layer of this thickness contributes to the signal observed in transmission.

\section{RESULTS AND DISCUSSION}

The observed SH intensity from the film-substrate interface as a function of the azimuthal angle of the sample is shown in Fig. 2 for a full $360^{\circ}$ rotation, and for four different combinations of polarizer and analyzer settings. The filled and empty points correspond to measurements at $\pm M$. The solid lines represent best fits to the data points using Eqs. (6)-(9). The obtained best fit parameters, normalized to $A$ (interface) $=1$, are listed in Table I. A very good fit is obtained, and no dependence on the direction of magnetization is observed, in agreement with the expected quadratic $\mathbf{M}$ 
TABLE I. Parameters of Eqs. (6)-(10) giving a best fit to the measured SHG rotational anisotropy. All values are normalized to $A($ interface $)=-1$.

\begin{tabular}{lcccc}
\hline \hline & & \multicolumn{3}{c}{ Reference } \\
Tensor comp. & Interface & Surface & $X X$ & $X Y$ \\
\hline$A$ & -1.00 & -0.11 & -0.06 & 0.09 \\
$B$ & 0.76 & 1.42 & 0.78 & 1.12 \\
$C$ & 2.22 & 0.56 & 0.31 & 0.44 \\
$D^{\mathrm{a}}$ & & $0.67^{\mathrm{b}}$ & 1.29 & 0.64 \\
\hline \hline
\end{tabular}

${ }^{3}$ Equations (6)-(9) are modified with a constant (isotropic and nonmagnetic) term $D$ to account for the observed nonmagnetic contribution to the signal, See Eq. (10).

'This nonmagnetic contribution is only used to fit the data for the $Y Y$ configuration.

dependence of $I(2 \omega)$. The $X X$ and $Y X$ curves show a fourfold symmetry with only four lobes because the values of the constant terms of Eqs. (6) and (8) are larger than the amplitude of the corresponding $\cos (4 \phi)$ terms. The $X Y$ and $Y Y$ curves show eightfold symmetry as expected in the absence of such constant terms.

Figure 3 shows the measured $\mathrm{SH}$ intensity originating from the film surface, obtained by illuminating the sample from the substrate side. For the configurations $X X, Y X$, and $X Y$ there is essentially no observable magnetic field dependence. The fourfold and eightfold symmetry of these curves is in accordance with Eqs. (6) and (8). The small lobes occurring in the $Y X$ configuration arise because the constant term in Eq. (8) now is smaller than the amplitude of the $\cos (4 \phi)$ term. In contrast, the $Y Y$ curve shows a pronounced

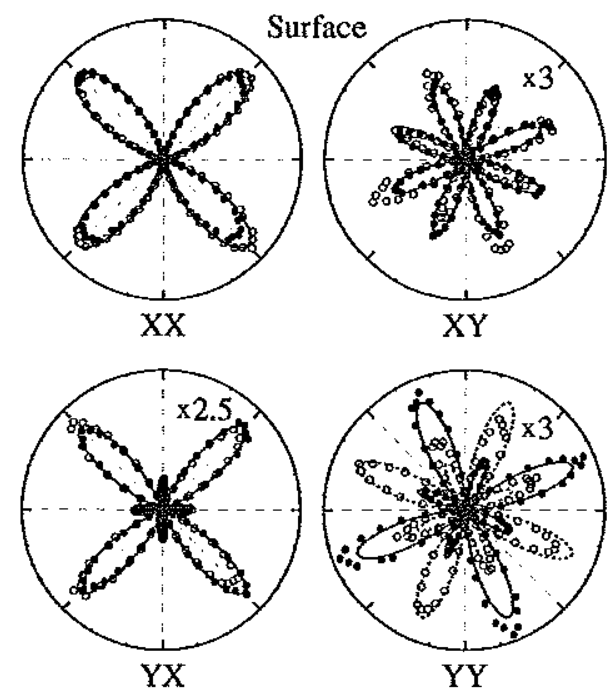

FIG. 3. Measured SHG rotational anisotropy $I(\phi)$ originating from the garnet film surface. The symbols below each plot denote the settings of polarizer and analyzer transmission axis, respectively. Open and filled circles represent measurements with the magnetic field in the $+Y$ and $-Y$ direction. The solid and dashed lines represent best fits to the data points. Only for the $Y Y$ configuration a dependence on the direction of the magnetic field is clearly seen.

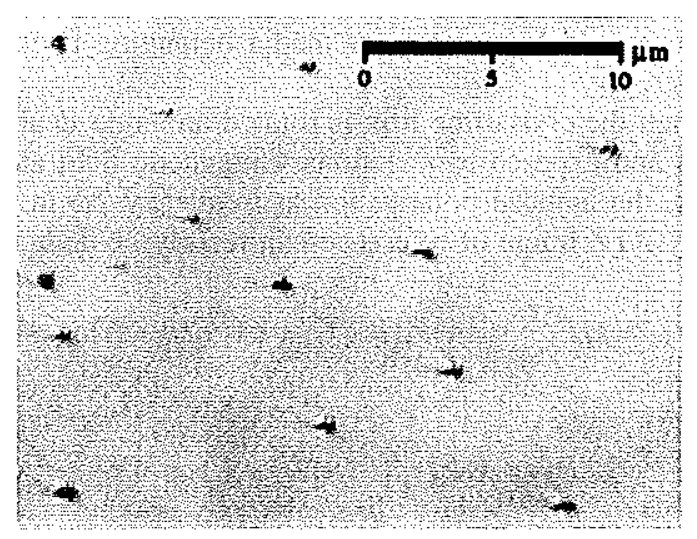

FIG. 4. Optical microscope image of the approximately $0.5 \mu \mathrm{m}$ inclusions observed on the garnet film surface. Their real size could be much smaller due to the diffraction limited resolution.

and unexpected dependence on the field direction. This must be attributed to interference between different sources of SHG which respond differently to the change in direction of the magnetic field. These could either be magnetic domains or clusters differently magnetized from the surrounding single crystalline film, or nonmagnetic sources such as inclusions or structural irregularities. Note that any dependence on the direction of $\mathbf{M}$ was never seen for the signal from the film-subsirate interface.

The data in Fig. $3(Y Y)$ can be described by Eq. (8) if a constant isotropic term $D$, independent of both $\mathrm{M}$ and $\phi$ is added

$$
P_{Y Y}^{\prime}=E_{Y}^{2} \frac{\epsilon_{0}}{4}[D-M(A+2 B+C) \sin 4 \phi]
$$

The curves then split for $\pm \mathbf{M}$ due to interference between the magnetization induced and the nonmagnetic contribution, as shown by the solid and dashed lines.

A distortion of the crystal symmetry could lead to crystallographic contributions to the $\mathrm{SHG}$, but it would be expected to have some angular dependence. Surface roughness or clusters could produce magnetic or nonmagnetic domains or grains with different properties from the rest of the material, and these might give an isotropic contribution to the SHG.

By studying the garnet film surface in an optical microscope we have found that, in fact, it has small inclusions scattered across the whole surface. The size of the dots in Fig. 4 appears to be approximately $0.5 \mu \mathrm{m}$, but their real size could be much smaller due to the diffraction limited resolution.

Ideally, the fit parameters for both surface and interface SHG should be the same. The fact that they are not may be explained by the different local environment. At the interface there is some strain due to lattice mismatch between film and substrate, which may have relaxed at the surface. In addition there could be gradients in the composition of the film, i.e., growth induced anisotropy.

The effects of the surface inclusions were further studied by measuring the dependence of the $\mathrm{SH}$ intensity on the in-plane field strength. With polarizer and analyzer axis oriented along the $Y$-direction the sample was rotated to an 


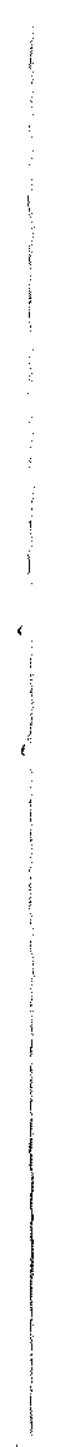


act as nonmagnetic sources of SHG. No direct evidence of domain pinning by these inclusions have been observed.

It was found that the magnetization at the film surface and interface saturates in the out-of-plane direction at different strengths of the applied field, and both higher than the field necessary to saturate the bulk. We conclude that the anisotropy leading to easy magnetization of the in-plane type is stronger at the interface than at the surface. A small four-fold in-plane magnetocrystalline anisotropy was also identified.

\section{ACKNOWLEDGMENTS}

Financial support by the Norwegian Research Council is gratefully acknowledged. The authors would also like to thank L. E. Helseth for providing the samples and for useful discussions. Part of this work was supported by the Stichting voor Fundamenteel Onderzoek der Materie (FOM), which is financially supported by the Nederlandse Organisatie voor Wetenschappelijk Onderzoek (NWO).
*Electronic address: fredrik.hansteen@phys.ntnu.no

${ }^{1}$ G. Winkler, Magnetic Gamets (Friedr. Vieweg, and Sohn, Braunschweig, Germany, 1981).

${ }^{2}$ Physics of Magnetic Gamets, edited by A. Paoletti, Enrico Fermi International School of Physics, Italian Physical Society (NorthHolland, Amsterdam, 1978), ISBN 0.444-85200-x.

${ }^{3}$ L. E. Helseth, Ph.D. thesis, University of Oslo, 2002.

${ }^{4}$ Magneto-Optical lmaging, edited by T. Johansen and D. Shantsev Vol. 142 of NATO Science Series II: Mathematics, Physics and Chemistry (Kluwer Academic, Dordrecht, 2004), ISBN 1-4020. 1997-1.

${ }^{5}$ L. Dorosinskii, M. Indenbom, I. Nikitenko, Y. Ossipyan, A. Polyanskii, and V. Vlaskovlasov, Physica C 203, 149 (1992).

${ }^{6}$ M. Koblischka and R. Wijngaarden, Supercond. Sci. Technol. 8, 199 (1995).

${ }^{7}$ P. Goa, H. Hauglin, M. Baziljevich, E. Il'yashenko, P. Gammel, and T. Johansen, Supercond. Sci. Technol. 14, 729 (2001).

${ }^{8}$ Y. Shen, The Principles of Nonlinear Optics (Wiley, New York, 1984).

${ }^{9}$ J. Reif, J. Zink, C. Schneider, and J. Kirschner, Phys. Rev. Lett. 67, 2878 (1991).

${ }^{10} \mathrm{H}$. Wierenga, W. de Jong, M. Prins, T. Rasing, R. Vollmer, A. Kirilyuk, H. Schwabe, and J. Kirschner, Phys. Rev. Lett. 74, 1462 (1995).

"M. Straub, R. VolImer, and J. Kirschner, Phys. Rev. Lett. 77, 743 (1996).

${ }^{12}$ T. Crawford, C. Rogers, T. Silva, and Y. Kim, Appl. Phys. Lett. 68,1573 (1996).
${ }^{13} \mathrm{~K}$. Bennemann, Nonlinear Optics in Metals, International Series of Monographs on Physics (Clarendon Press, Oxford, 1998).

${ }^{14}$ V. Pavlov, R. Pisarev, M. Fiebig, and D. Fröhlich, Phys. Solid State 45, 662 (2003).

${ }^{15}$ M. Fiebig, D. Fröhlich, K. Kohn, S. Leute, T. Lottermoser, V. Pavlov, and R. Pisarev, Phys. Rev. Lett. 84, 5620 (2000).

${ }^{16}$ M. Fiebig, D. Fröhlich, B. Krichevtsov, and R. Pisarev, Phys. Rev. Lett. 73, 2127 (1994).

${ }^{17}$ R. Pisarev, B. Krichevtsov, V. Gridnev, V. Klin, D. Fröhlich, and C. Pahlke-Lerch, J. Phys.: Condens. Matter 5, 8621 (1993).

${ }^{18}$ V. Gridnev, V. Pavlov, R. Pisarev, A. Kirilyuk, and T. Rasing, Phys. Rev. B 63, 184407 (2001).

${ }^{19}$ V. Pavlov, R. Pisarev, A. Kirilyuk, and T. Rasing, Phys. Rev. Lett. 78, 2004 (1997).

${ }^{20}$ R. Stolle, K. Veenstra, F. Manders, T. Rasing, H. van den Berg, and N. Persat, Phys. Rev. B 55, R4925 (1997).

${ }^{21}$ R. R. Birss, Symmetry and Magnetism, Series of Monographs on Selected Topics in Solid State Physics, 2nd ed. (North-Holland, Amsterdam, 1966).

${ }^{22}$ D. L. Wood and K. Nassau, Appl. Opt. 29, 3704 (1990).

${ }^{23}$ F. Hansteen, L. E. Helseth, T. H. Johansen, O. Hunderi, A. Kirilyuk, and T. Rasing, Thin Solid Films 455-456C, 429 (2004).

${ }^{24}$ R. Chang, J. Ducuing, and N. Bloembergen, Phys. Rev. Lett. 15, $6(1965)$.

${ }^{25}$ K. Veenstra, A. Petukhov, A. de Boer, and T. Rasing, Phys. Rev. B 58, R16020 (1998).

${ }^{26}$ L. Helseth, R. Hansen, E. Il'yashenko, M. Baziljevich, and T. Johansen, Phys. Rev. B 64, 174406 (2001). 
$X Y$ curve splits. The fitting parameters are listed in Table I. Note that the value of the coefficients $A, B$, and $C$ are the same as those found by fitting the surface signal in Fig. 3, only scaled by a factor 0.55 and 0.79 for the $X X$ and $Y X$ configurations, respectively. The reason for this scaling is that the polymer film absorbs some of the SH light. The different magnitude of the constant contribution $D$ for the $X X$ and $X Y$ configurations is due to the strongly anisotropic second-order susceptibility of the stationary polymer film, i.e. $\chi_{x x x} \neq \chi_{y x x}$.

Dispersion in the substrate prevents the same experiment to be conducted with the SHG from the interface, however, the problem may be overcome by analyzing the spectrum of the signal. ${ }^{25}$

\section{B. Polar magnetic field dependence of the SHG}

In order to further investigate the surface and interface magnetic properties in comparison to those of the bulk, the magnetization-induced SHG signal, and the (bulk) linear Faraday rotation were studied as function of an out-of-plane magnetic field. The sample was placed in a strong polar magnetic field, i.e., along the $Z$-direction, and rotated to an azimuthal angle giving a peak in the observed SH intensity. The intensity of the SH light and the linear Faraday rotation of the fundamental beam were then measured simultaneously as a function of the polar field strength up to $600 \mathrm{kA} / \mathrm{m}$. This was done for light incident from both the substrate and the film side of the sample.

In both cases the strongest SHG signal was observed close to zero polar magnetic field. The spontaneous magnetization of the garnet film is then in-plane. As the magnetization is gradually forced out-of-plane by the polar field, the SHG signal decreases and eventually vanishes (reaches level of PMT background noise) as the magnetization is saturated in the out-of-plane direction. This is shown in Fig. 7. The figure also shows the simultaneously measured Faraday rotation of the fundamental beam, corrected for the contribution of the paramagnetic GGG substrate. The Faraday rotation appears to saturate at a clearly lower value of the magnetic field than the SH signal from both surface and interface, indicating that the bulk magnetization saturates in the out-of-plane direction before the surface and interface magnetizations. Furthermore, it is very interesting to note the different saturation magnetic fields of the SH signal from the surface and interface. A significantly stronger field is required to saturate the interface magnetization in the out-ofplane direction, again demonstrating that the anisotropy at the interface, leading to an easy magnetization of the inplane type, is relaxed at the surface.

The noise and sudden drop in the SH intensity near zero field can be explained by the random in-plane orientation of the magnetization. As demonstrated by the rotational anisotropy measurements, the orientation of the in-plane magnetization with respect to the crystal lattice has a large influence on the SHG response.

This experiment demonstrates the applicability of SHG to probe surface and interface magnetic properties. In addition, the results again confirm the dominantly magnetic origin of

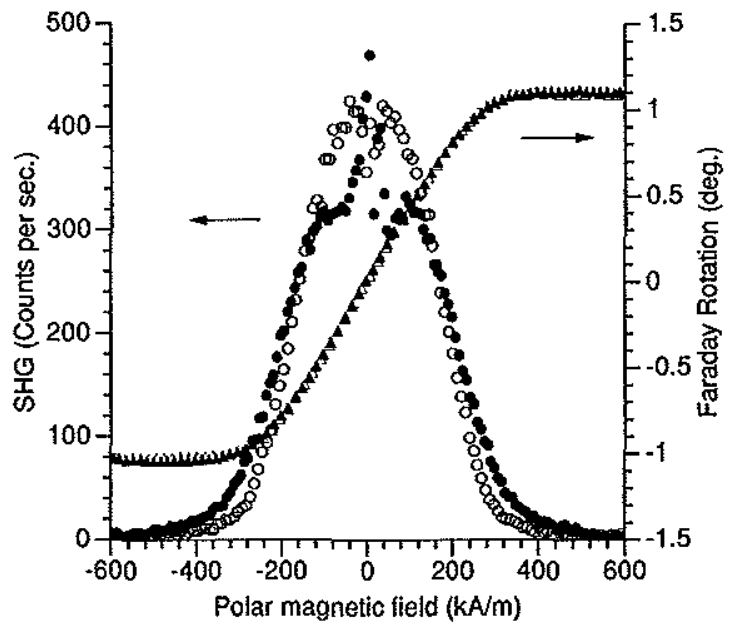

FIG. 7. Simultaneously measured SHG intensity and Faraday rotation of the fundamental beam as a function of the applied polar magnetic field. Results obtained with the garnet sample illuminated from the substrate side are shown by empty symbols, while the filled symbols represent measurements with light incident from the film surface side. The data have been averaged over measurements taken at increasing and decreasing magnetic ficld.

the observed SHG, and also demonstrate that the SHG effectively can be switched off using a polar magnetic field. This could find technological applications, for instance as a switchable element in lasers. The field strength required to completely switch off the SHG, that is to orient the magnetization in the out-of-plane direction, depends strongly on the chemical composition of the garnet film. ${ }^{26}$ We have verified for other garnet films grown on the same substrate orientation, but with higher concentrations of gallium, that the Faraday rotation saturates and the SHG signal vanishes at lower magnetic fields. The sensitivity can thus be tuned by altering the composition of the films. Modulation or switching of the SHG can also be achieved by varying the direction of the in-plane magnetization which requires much smaller magnetic fields.

\section{CONCLUSIONS}

We have shown, for thin garnet films grown on (100) oriented GGG substrates, that magnetization-induced SHG can serve as a powerful interface selective probe of the local magnetic structure. Since the space inversion symmetry in this case is broken throughout the whole film, the interface sensitivity is not due to symmetry breaking by the interfaces, but stems from the fact that the onset of strong optical absorption in the garnet films is conveniently located at about $2.3 \mathrm{eV}$, in-between the fundamental and second hamonic frequencies.

We have experimentally confirmed that the observed SHG from our film-substrate interface is of purely magnetic origin, as predicted by electromagnetic theory in the electric dipole approximation. The surface signal is dominantly of magnetic origin but also has a small nonmagnetic component. We have found that this is due to small grainlike inclusions in the surface of these LPE grown garnet films which 

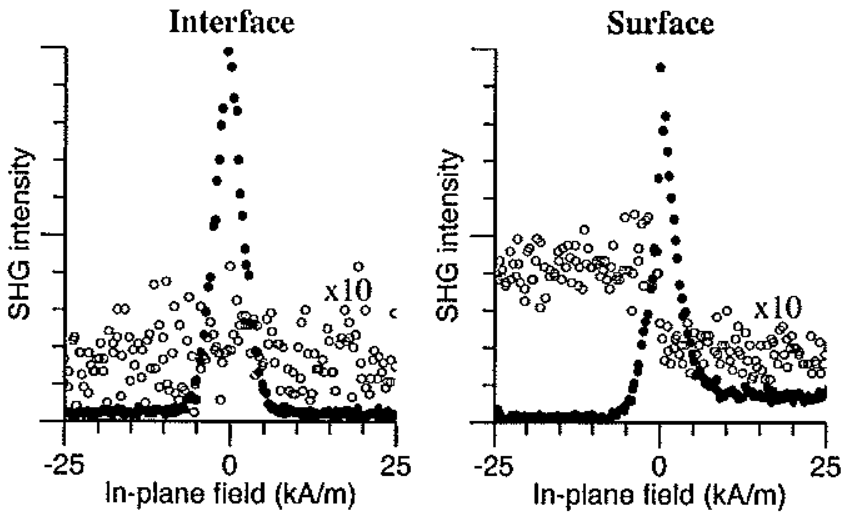

FIG. 5. Dependence of the SH intensity on the strength of the applied transverse (in-plane) magnetic field. Empty and filled points correspond to measurements at two neighboring minima in the $Y Y$ configuration, i.e., at $\phi^{\prime}$ and $\phi^{\prime}+45^{\circ}$, respectively. The very different behavior near zero applied field is due to a small in-plane magnetocrystalline anisotropy which causes different switching behavior of the magnetization depending on its orientation with respect to the crystal axes. Interference between magnetic and nonmagnetic sources of SHG can be seen as steps in the SH intensity originating from the film surface, while the interface signal is of purely magnetic origin.

angle $\phi=\phi^{\prime}$ giving a minimum in the SH signal. For this $Y Y$-configuration there are eight equally spaced minima, as shown by the SHG rotational anisotropy in Figs. 2 and 3 . The SH intensity was recorded as a function of the strength of the applied magnetic field, and the results are shown in Fig. 5 for both the surface and the interface signal. The empty points represent measurements at $\phi=\phi^{\prime}$, which is also the same orientation at which the $X X$-configuration gives a minimum signal, while the filled points were measured at a neighboring minimum, $\phi=\phi^{\prime}+45^{\circ}$. For these two orientations of the sample, the behavior of the SH intensity near zero applied field is very different. At $\phi=\phi^{\prime}$ the interface signal does not show any dependence on the strength of the applied field. The constant SHG signal even near zero applied field indicates that the magnetization simply flips $180^{\circ}$ as the field changes polarity. In contrast, the SH intensity increases strongly near zero applied field for the $\phi=\phi^{\prime}$ $+45^{\circ}$ orientation of the sample. In addition to the fact that the easy direction of magnetization of this film is of the in-plane type, there also exists a small fourfold magnetocrystalline anisotropy in the film plane. Because of this magnetocrystalline anisotropy the magnetization does not simply flip $180^{\circ}$, but appears to first rotate $90^{\circ}$ to an equivalent and preferred in-plane orientation. This conclusion is supported by the fact that the peak intensity at zero field is equal to the maximum intensity measured for the rotational anisotropy in the $X X$ configuration (Figs. 2 and 3 ).

It is also evident from Fig. 5 that the surface SHG signal is composed of a magnetic and a nonmagnetic source. The interference between these gives rise to the different signal levels for $\pm \mathbf{M}$ as described by Eq. (4). Also for the surface signal the influence of the in-plane magnetocrystalline anisotropy on the switching mechanism is evident. When oriented at $\phi=\phi^{\prime}$ there is simply a step in the SHG intensity due to

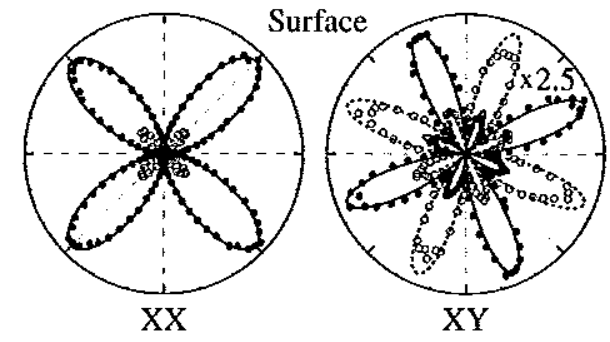

FIG. 6. SHG rotational anisotropy measurement of the surface of the garnet film with a stationary external polymer reference. The signal for $\pm \mathbf{M}$ can be seen to split due to interference between the magnetization induced SHG in the garnet film and the SHG in the polymer film.

the phase change of $\pi$ in the magnetization-induced contribution as M changes sign. At $\phi=\phi^{\prime}+45^{\circ}$ the same mechanism of switching as described for the interface can be seen. Interference between nonmagnetic and magnetic sources of SHG is also clearly seen as a difference in signal levels for $\pm \mathrm{M}$.

The experimental data in Fig. 5 show no evidence of domain pinning by the surface inclusions. The dependence of the SH intensity on the field was measured at in-plane fields up to $300 \mathrm{kA} / \mathrm{m}$, but remained constant at the levels shown in the figure. It is not clear what the inclusions are but apparently they cause a nonmagnetic contribution to the SHG from the film surface, most clearly seen in the $Y Y$ configuration. Note that a similar effect, though much weaker is observed in the $X Y$ polarization combination.

\section{A. Phase-sensitive measurements of SHG}

In accordance with the experimental results of Fig. 3, configurations $X X, X Y$, and $Y X$, the radiated intensity [Eq. (4)] appears not to be sensitive to the phase change of $\pi$ in the induced polarization at $2 \omega$ [Eqs. (6)-(9)] that occurs when the magnetization is reversed, $\mathbf{M} \rightarrow-\mathbf{M}$. To confirm the existence of this phase shift the $\mathrm{SH}$ wave from the magnetic garnet sample was made to interfere with a SH wave generated in a nonmagnetic "reference" sample. ${ }^{20,24}$ By placing a glass substrate coated with a polymer film having large second-order optical nonlinear coefficients just after the garnet film we achieve spatial overlap of the $\mathrm{SH}$ waves from the two sources. As long as no strongly dispersive elements are inserted between the garnet film and the reference sample, the fundamental pulse and the SH pulse will also overlap temporally as they reach the reference. SH radiation generated in the polymer film will then interfere with the SH light from the garnet film. This can be observed as a change in detected intensity upon reversal of the direction of magnetization, because the interference term in Eq. (4) changes sign.

This can be modeled by Eqs. (6)-(9) by adding a constant isotropic term $D$, as was done in Eq. (10). The rotational anisotropy of the total SH intensity is shown in Fig. 6 for $\pm M$. As the direction of the magnetic field is reversed, the SHG from the garnet film experiences a phase shift of $\pi$ and the interference term of Eq. (4) changes sign. For the $X X$ configuration the intensity is reduced significantly while the 